Journal for ImmunoTherapy of Cancer

\section{Infiltrating pattern and prognostic value of tertiary lymphoid structures in resected non-functional pancreatic neuroendocrine tumors}

To cite: Zhang W-H, Wang W-Q, Han $X$, et al. Infiltrating pattern and prognostic value of tertiary lymphoid structures in resected non-functional pancreatic neuroendocrine tumors. Journal for ImmunoTherapy of Cancer 2020;8:e001188. doi:10.1136/ jitc-2020-001188

- Additional material is published online only. To view, please visit the journal online (http://dx.doi.org/10.1136/jitc2020-001188).

W-HZ, XH and H-LG contributed equally.

Accepted 21 September 2020

Check for updates

(C) Author(s) (or their employer(s)) 2020. Re-use permitted under CC BY-NC. No commercial re-use. See rights and permissions. Published by BMJ.

For numbered affiliations see end of article.

\section{Correspondence to}

Dr Wen-Quan Wang;

wangwenquan@fudanpci.org

Professor Xian-Jun Yu; yuxianjun@fudanpci.org

Professor Liang Liu; liuliang@fudanpci.org

\section{ABSTRACT}

Background Tertiary lymphoid structures (TLS) are associated with favorable survival and play a critical role in most solid tumors. However, investigations of TLS are lacking in patients with grade 1 or grade 2 (G1/G2) non-functional pancreatic neuroendocrine tumors (NFPanNETs). This study aimed to investigate the presence, cellular composition, association with tumor-infiltrating immune cells, and prognostic value of TLS in G1/G2 NFPanNETs.

Methods Tumor tissues from a 182-patient Fudan cohort and a 125-patient external validation set were assessed by H\&E staining, immunohistochemistry, and/or multispectral fluorescent immunohistochemistry.

Results TLS were identified in more than one-third of patients with G1/G2 NF-PanNETs and were located peritumorally, either just outside the tumor tissue or in the stromal area. TLS were mainly composed of B-cell follicles with germinal centers and T-cell zones with dendritic cells. Kaplan-Meier analyses showed that the presence of TLS correlated with both longer recurrence-free survival (RFS, $p<0.001$ ) and overall survival (OS, $p=0.001$ ), but the number of TLS had no prognostic significance. Multivariate Cox-regression analyses demonstrated that the presence of TLS, WHO classification, and 8th edition American Joint Committee on Cancer (AJCC ${ }^{\text {th }}$ ) tumor-node-metastasis (TNM) stage were independent prognostic factors for RFS ( $p=0.004, p=0.001$, and $p<0.001$, respectively) and $0 S$ $(p=0.009, p=0.008$, and $p=0.019$, respectively). These results were confirmed using an external validation set. Finally, a nomogram incorporating the presence of TLS was constructed to predict the probability of 5-year RFS of resected G1/G2 NF-PanNETs, which improved on the current WHO classification and AJCC ${ }^{8 \text { th }}$ TNM stage. Conclusions The presence of TLS is an independent and favorable predictor of resected G1/G2 NF-PanNETs, which may play a role in cancer immunobiology.

\section{INTRODUCTION}

Pancreatic neuroendocrine tumors (PanNETs) are a rare, heterogeneous group of tumors arising from the neuroendocrine system and are classified as functional or non-functional (NF-PanNETs). ${ }^{1}$ NF-PanNETs account for $60 \%-90 \%$ of PanNETs, tend not to secrete biologically active hormones or neuropeptides and have no characteristic symptoms. $^{2-4}$ More than $90 \%$ of resected PanNETs are grade 1 or grade 2 (G1/G2) tumors, ${ }^{5}$ with a Ki67 index $<20 \% .{ }^{6}$ However, as heterogeneous tumors, variable genetic components and clinical behaviors of G1/ G2 NF-PanNETs shape tumor fate. It has been difficult to predict recurrence or overall survival (OS) and thus, a better understanding of G1/G2 NF-PanNETs will inform clinical decisions. ${ }^{7}$

The tumor-immune microenvironment provides a functional context affecting tumor growth and progression. Recent reviews have described the tumor-immune microenvironment and delineated the immune components in gastroenteropancreatic NETs. ${ }^{8-12}$ Tertiary lymphoid structures (TLS) are an important element of the tumor-immune microenvironment. These structures are ectopic lymphoid aggregates detected as intratumoral and peritumoral immune cell clusters and represent sites of lymphoid neogenesis in most solid tumors. ${ }^{13} 14$ Similar to secondary lymphoid organs (SLO), particularly in morphology and cellular composition, ${ }^{15} 16$ TLS may play an important role in recruiting lymphocytes and promoting an efficacious immune microenvironment. ${ }^{17}$ High densities of TLS are associated with good clinical outcomes in pancreatic, ${ }^{18}$ colorectal, gastric, ${ }^{19}$ lung, ${ }^{20}$ and breast cancer, ${ }^{21}{ }^{22}$ and oral squamous cell carcinoma, indicating TLS might be a predictive and prognostic factor. Further, TLS might form a compartment that generates memory $\mathrm{T}$ cells and $\mathrm{B}$ cells, thus aiding immune responses against tumors. ${ }^{14}$ 
However, TLS have also been suggested to act as microniches for cancer progenitor cells, and their presence is correlated with an increased risk of late recurrence in hepatocellular carcinoma. ${ }^{23}$

The prognostic role of TLS in neuroendocrine neoplasms remains largely unexplored. The presence of TLS does not appear to impact prognosis in small bowel NETs. ${ }^{11}$ Further, the prognostic role of TLS remains unexplored in G1/G2 NF-PanNETs. Here, we identified the presence of TLS in G1/G2 NF-PanNETs, defined the cellular composition of these TLS and their association with tumor-infiltrating immune cells as well as the prognostic significance of TLS in a large cohort of NF-PanNETs.

\section{METHODS \\ Patient population}

This study included two independent patient cohorts. The Fudan cohort contained 182 patients who underwent surgery for histologically confirmed G1/G2 NF-PanNETs at Fudan University Shanghai Cancer Center (FUSCC), between 2006 and 2018. The external validation set comprised 125 patients with G1/G2 NF-PanNETs who underwent a pancreatectomy at an external hospital with pathology consultations performed at our institute. To study TLS in heterogeneous pancreatic neuroendocrine neoplasms (PanNENs), we enrolled 26 patients with G1/G2 functional PanNETs (22 insulinomas and 4 gastrinomas), 22 patients with G3 NF-PanNETs, and 18 patients with poorly differentiated pancreatic neuroendocrine carcinomas (PanNECs). All 66 patients underwent surgery and their diagnosis was histologically confirmed at FUSCC. Surgical history, clinicopathological parameters, and follow-up information were collected retrospectively from our medical record database. Patients were followed up with every 3 months for the first 2 years after surgery. Then, patients were followed up every 6 months until recurrence or death. At each follow-up, patients underwent a physical examination, laboratory investigation and contrast-enhanced CT or MRI of the chest/ abdomen/pelvis. Recurrence-free survival (RFS) was defined as the time from the date of surgery to the date of recurrence. OS was defined as the time from the date of surgery to the date of either death or the last follow-up. Tumors were restaged and regraded based on the 8th edition American Joint Committee on Cancer (AJCC ${ }^{8 \text { th }}$ ) tumor-node-metastasis (TNM) staging system and 2017 WHO classification.

\section{Evaluation of TLS}

Formalin-fixed, paraffin-embedded (FFPE) specimens from each patient in the 182-patient Fudan cohort and 66 patients with PanNENs were prepared in $5 \mu \mathrm{m}$ thick sections for H\&E staining. ${ }^{24} \mathrm{H} \& \mathrm{E}$-stained sections of the 125-patient external validation set were borrowed from the pathology department at our institute. To further assess lymphocytic organization in G1/G2 NF-PanNETs, immunohistochemistry (IHC) staining was carried out as previously described,${ }^{25-28}$ using the 182 serial sections from the Fudan cohort. IHC staining was performed to identify helper T cells (CD4, ab133616, 1:200, Abcam), cytotoxic T cells (CD8, ab172729, 1:300, Abcam), regulatory T cells (Tregs, FOXP3, ab20034, 1:800, Abcam), memory T cells (CD45RO, ab23, 1:1,000, Abcam), B cells (CD20, 24828-1-AP, 1:5,000, Proteintech), dendritic cells (DCs, CD11c, ab52632, 1:500, Abcam), natural killer cells (NK cells, anti-NCR1, ab224703, 1:1,000, Abcam), and tumor-associated macrophages (TAMs, CD68, ab213363, 1:4,000, Abcam).

TLS were identified on full-face H\&E slides of 307 tumors from the Fudan cohort, the external validation set, and the 26 G1/G2 functional PanNETs, 22 G3 NF-PanNETs, and 18 PanNECs tissues. Slides were reviewed by two independent observers (Dr Dan Huang and Dr Hai-Ying Zeng) for the presence and the location of TLS. When TLS were identified, the numbers of tumor-infiltrating immune cells outside the TLS were counted in G1/G2 NF-PanNET tissue by evaluating five random high-power fields $(\times 200)$. Data are presented as the mean \pm SD.

\section{Multispectral fluorescent IHC}

Five FFPE slides from specimens, with observed TLS in the H\&E-stained sections, were subjected to multispectral immunohistochemical (mIHC) staining ${ }^{29} 30$ using the Opal color kit (PerkinElmer, Hopkinton, Massachusetts, USA), according to the manufacturer's instructions, to provide simultaneous detection of adaptive immune system cell types, such as $\mathrm{CD} 4^{+} \mathrm{T}$ cells, $\mathrm{CD}^{+} \mathrm{T}$ cells, $\mathrm{CD}^{+} 0^{+} \mathrm{B}$ cells, and $\mathrm{CD} 45 \mathrm{RO}^{+}$memory T cells. Cell nuclei were stained with DAPI. Multiplexed color slides were imaged with a PerkinElmer Vectra automated multispectral microscope at $100 \times$ magnification and analyzed using PerkinElmer inForm Analysis Software.

\section{Nomogram construction}

Nomogram construction used a multivariate Cox model for RFS, based on TLS, WHO classification, and AJCG ${ }^{8 \text { th }}$ TNM stage, and was fit to the Fudan cohort. Validation was performed using bootstrap resampling; external validation was performed using the 125-patient external validation set. ${ }^{6}$ Model performance was evaluated using the concordance index (c-index) and calibration curve. ${ }^{31}$ The c-index was calculated for the WHO classification and AJCC $^{\text {th }}$ TNM stage, and the two indices were compared with the c-index from the nomogram.

\section{Statistical analyses}

Statistical analyses were performed using SPSS (V.25.0, IBM, Armonk, New York, USA) and Stata (V.15.0, Stata Corp., Houston, Texas, USA). Nomograms were constructed using R V.3.6.3 (R Foundation for Statistical Computing, Vienna, Austria). Continuous variables were compared by non-parametric tests or Student's t-test. Categorical variables were compared by Pearson's $\mathrm{X}^{2}$ test or Fisher's test. The Kaplan-Meier method and 
Table 1 Demographics and clinical characteristics of the Fudan cohort and external validation set of patients with G1/ G2 NF-PanNETs

\begin{tabular}{|c|c|c|c|}
\hline \multirow[b]{2}{*}{ Characteristics } & $\begin{array}{l}\text { Fudan cohort } \\
(\mathrm{n}=182)\end{array}$ & $\begin{array}{l}\text { External } \\
\text { validation set } \\
(n=125)\end{array}$ & \multirow[b]{2}{*}{$P$ value } \\
\hline & No. (\%) & No. (\%) & \\
\hline Sex & & & 0.252 \\
\hline Male & $87(47.8)$ & $60(48.0)$ & \\
\hline Female & 95 (52.2) & $65(52.0)$ & \\
\hline Age (years) (median) & 53 & 54 & 0.940 \\
\hline Tumor size (mm) & & & 0.490 \\
\hline Mean (SD) & $32.9(2.0)$ & $34.7(2.4)$ & \\
\hline Median (range) & $29.0(6.0-95.0)$ & $30.0(6.0-137.0)$ & \\
\hline Location & & & 0.659 \\
\hline Head & $76(41.8)$ & $47(37.6)$ & \\
\hline Others & $106(58.2)$ & $78(62.4)$ & \\
\hline Lymph node positive & & & 0.741 \\
\hline Yes & $36(19.8)$ & $27(21.6)$ & \\
\hline No & $146(80.2)$ & $98(78.4)$ & \\
\hline Vascular invasion & & & 0.578 \\
\hline Yes & $45(24.7)$ & $33(26.4)$ & \\
\hline No & $137(75.3)$ & $92(73.6)$ & \\
\hline Perineural invasion & & & 0.980 \\
\hline Yes & $39(21.4)$ & $27(21.6)$ & \\
\hline No & $143(78.6)$ & $98(78.4)$ & \\
\hline AJCC $^{8 \text { th }}$ TNM stage & & & 0.819 \\
\hline I & $72(39.6)$ & $48(38.4)$ & \\
\hline II & $74(40.7)$ & $50(40.0)$ & \\
\hline III & $36(19.8)$ & 27 (21.6) & \\
\hline WHO classification & & & 0.704 \\
\hline G1 & $120(65.9)$ & $78(62.4)$ & \\
\hline G2 & $62(34.1)$ & $47(37.6)$ & \\
\hline Presence of TLS & & & 0.223 \\
\hline Yes & $65(35.7)$ & $46(36.8)$ & \\
\hline No & $117(64.3)$ & 79 (63.2) & \\
\hline
\end{tabular}

$\mathrm{AJCC}^{8 \text { th }}$, 8th edition American Joint Committee on Cancer; G1/ G2, grade 1/grade 2; NF-PanNETs, non-functional pancreatic neuroendocrine tumors; TLS, tertiary lymphoid structures; TNM, tumor-node-metastasis.

log-rank test were used to estimate statistical differences in survival. Cox-regression analyses were performed to identify independent prognostic factors of RFS and OS. Variables significantly associated with RFS or OS in the univariate analysis were included in the multivariate analysis. Two-sided $p$ values of $<0.05$ were considered statistically significant.
RESULTS

\section{Clinicopathological characteristics}

The clinicopathological characteristics of two patient cohorts are summarized in table 1. In the Fudan cohort, the 1-year, 3-year, and 5-year recurrence rates were $12.6 \%$, $25.3 \%$, and $28.0 \%$, respectively; and the 1-year, 3-year, and 5-year survival rates were $100 \%, 96.7 \%$, and $90.7 \%$, respectively. More than half of the patients were women $(52.2 \%)$. The median age was 53.0 years (range, 25-82 years). The median tumor size was $29.0 \mathrm{~mm}$ (range, $6.0-95.0 \mathrm{~mm}$ ). Positive lymph nodes were present in 36 patients $(19.8 \%)$. Vascular invasion and perineural invasion were observed in $45(24.7 \%)$ and 39 cases (21.4\%), respectively. The percentages of patients with $\mathrm{AJCC}^{8 \mathrm{th}}$ TNM stages I, II, and III were $39.6 \%, 40.7 \%$, and $19.8 \%$, respectively, and $\mathrm{WHO}$ grades 1 and 2 were $65.9 \%$ and $34.1 \%$, respectively.

\section{Characteristics of TLS in G1/G2 NF-PanNETs}

We defined the presence, location and cellular composition of TLS and related these to the clinicopathological characteristics of G1/G2 NF-PanNETs. TLS were located peritumorally, either just outside the tumor tissue or in the stroma. No TLS were observed in peritumoral normal pancreatic tissues. TLS were variable in size and shape, with most being oval or irregular (online supplemental figure S1), and were observed in $35.7 \%$ and $36.8 \%$ of G1/ G2 NF-PanNETs in the Fudan cohort and external validation set, respectively.

To characterize the cellular composition of TLS, we used IHC to identify $\mathrm{CD} 4^{+} \mathrm{T}$ cells, $\mathrm{CD} 8^{+} \mathrm{T}$ cells, $\mathrm{CD} 20^{+} \mathrm{B}$ cells, CD11 $c^{+}$DCs, CD45RO ${ }^{+}$memory T cells, anti-NCR1 ${ }^{+}$NK cells, $\mathrm{FOXP}^{+}$Tregs, and $\mathrm{CD} 68^{+}$TAMs in serial sections (figure $1 \mathrm{~A}-\mathrm{H}$ ). $\mathrm{CD}^{+}$and $\mathrm{CD}^{+} \mathrm{T}$ cells were primarily in the parafollicular cortex, with $\mathrm{CD} 4^{+} \mathrm{T}$ cells showing more infiltration than $\mathrm{CD}^{+}{ }^{+} \mathrm{T}$ cells. Most $\mathrm{CD} 20^{+} \mathrm{B}$ cells were in the center of the follicle, whereas $\mathrm{CD} 11 \mathrm{c}^{+}$DCs and $\mathrm{CD}_{45} \mathrm{RO}^{+}$memory $\mathrm{T}$ cells were located primarily within the T-cell zone, with some dispersion into the center of the follicle. Only sporadic anti-NCR $1^{+} \mathrm{NK}$ cells were identified, and $\mathrm{CD} 68^{+}$TAMs were rarely observed. $\mathrm{FOXP}^{+}$ Tregs were rare in both TLS and tumor tissues.

Next, we used mIHC to simultaneously detect CD45RO ${ }^{+}$, $\mathrm{CD}^{2} 0^{+}, \mathrm{CD}^{+}$, and $\mathrm{CD} 8^{+}$cells in G1/G2 NF-PanNET tissues and define their relationship to TLS and the tumor microenvironment (figure 2B-F). We focused on these adaptive immune cells because they were the major components of TLS based on IHC. The mIHC data reflected the IHC results, with $\mathrm{CD}_{4} 5 \mathrm{RO}^{+}$memory $\mathrm{T}$ cells showing more infiltration and greater dispersion into the center of the follicle in mIHC. Quantification of the cell types in the mIHC images showed that $\mathrm{CD}_{4} 5 \mathrm{RO}^{+}$memory $\mathrm{T}$ cells were the most common immune cells in TLS, followed by $\mathrm{CD} 20^{+} \mathrm{B}$ cells, $\mathrm{CD} 4^{+} \mathrm{T}$ cells, and $\mathrm{CD} 8^{+} \mathrm{T}$ cells $(39.35 \%$, $36.61 \%, 20.05 \%$, and $3.99 \%$, respectively, figure $2 \mathrm{G}-\mathrm{H}$ ). $\mathrm{CD} 20^{+} \mathrm{B}$ cells tended to be located in the center of the follicle whereas $\mathrm{CD}^{+} \mathrm{T}$ cells, $\mathrm{CD}^{+} \mathrm{T}$ cells, and 

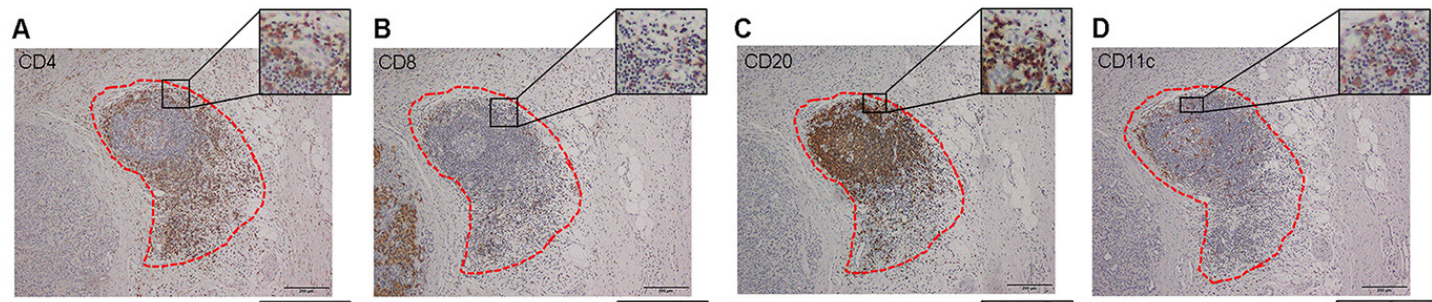

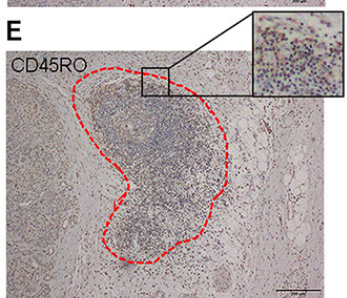

I

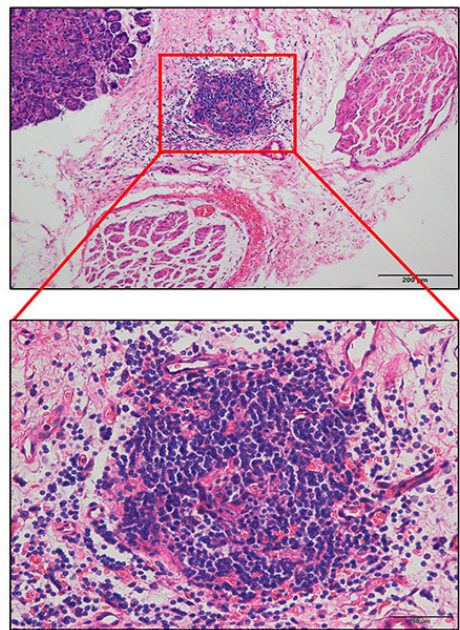

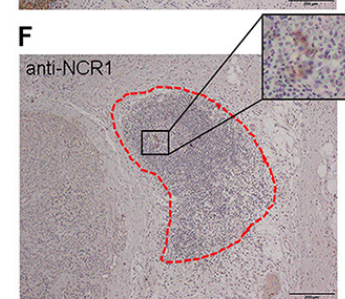

J

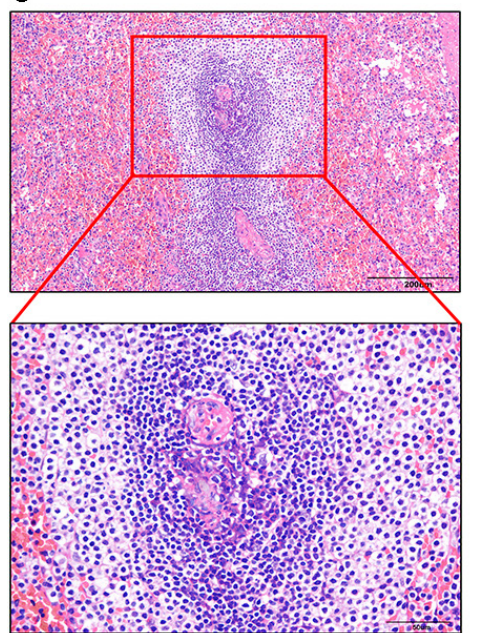

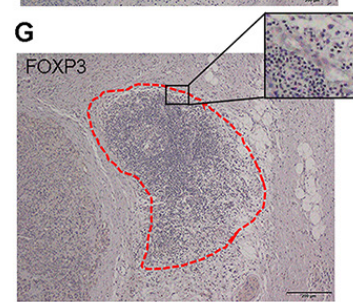

$\mathrm{K}$

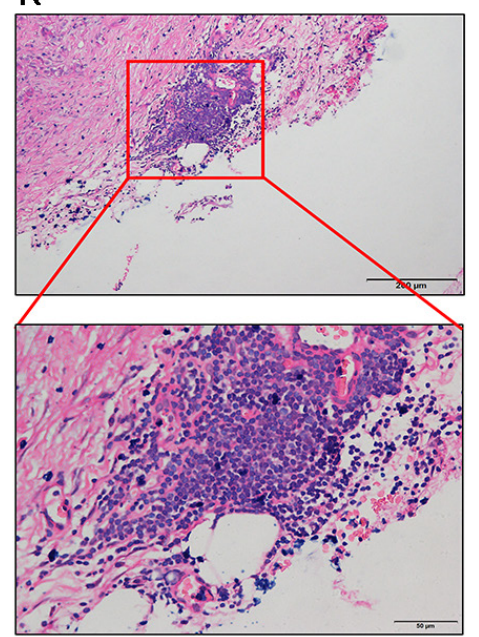

Figure 1 Immunohistochemistry and histology of tertiary lymphoid structures (TLS). (A-H) Cellular composition of TLS. (A) $\mathrm{CD4}^{+} \mathrm{T}$ cells. (B) $\mathrm{CD} 8^{+} \mathrm{T}$ cells. (C) $\mathrm{CD} 2 \mathrm{O}^{+} \mathrm{B}$ cells. (D) CD11c ${ }^{+}$dendritic cells. (E) CD45RO ${ }^{+}$memory T cells. (F) anti-NCR1 ${ }^{+}$natural killer cells. (G) FOXP3 $3^{+}$regulatory T cells. (H) CD68 tumor-associated macrophages. Magnification: 100x. (I-K) TLS stained with H\&E. The presence of TLS in NF-PanNETs (I), functional PanNETs (J), and PanNECs (K). Top row, magnification: 100x. Bottom row, magnification: 400x. NF-PanNETs, non-functional PanNETs; PanNECs, pancreatic neuroendocrine carcinomas; PanNETs, pancreatic neuroendocrine tumors.

$\mathrm{CD}_{45} \mathrm{RO}^{+}$memory $\mathrm{T}$ cells were primarily located in the parafollicular zone.

Patients with G1/G2 NF-PanNETs containing TLS associated with better clinical outcomes based on clinicopathological characteristics, such as negative lymph node, absence of vascular and perineural invasion, lower TNM stage (I, II), and lower WHO grade (G1), although statistical significance was observed only for vascular invasion. Patients without vascular invasion showed a higher percentage of TLS versus patients with vascular invasion $(40.88 \%$ vs $20 \%, \mathrm{p}=0.011)$. There was no significant difference in the percentage of TLS between patients with negative or positive lymph nodes $(36.30 \%$ vs $33.33 \%, p=0.739)$. Likewise, there was no significant difference in the percentage of TLS between patients with or without perineural invasion $(30.77 \%$ vs $37.06 \%, \mathrm{p}=0.467$ ). Additionally, statistical significance was not reached when comparing the percentage of TLS between patients with AJCC ${ }^{\text {th }}$ TNM stage I/II or III tumors $(36.99 \%$ vs $30.56 \%, p=0.471)$, and patients with WHO G1 or G2 tumors ( $40 \%$ vs $27.42 \%$, $\mathrm{p}=0.093$, online supplemental figure S2A).

\section{Characteristics of TLS in G1/G2 functional PanNETs, G3 NF- PanNETs, and PanNECs}

To better characterize TLS in heterogeneous PanNENs, we examined 26G1/G2 functional PanNETs, 22G3 NF-PanNETs, and 18 PanNECs. TLS were located peritumorally, varied in shape and size and had similar cellular composition (figure 1I-K). TLS were observed in $38.46 \%$ of G1/G2 functional PanNETs, 27.28\% of G3 NF-PanNETs, and $11.11 \%$ of PanNECs. Although the percentage of TLS in G1/G2 NF-PanNETs was lower than in G1/G2 functional PanNETs and higher than in G3 NF-PanNETs, these differences did not reach statistical significance. However, the percentage of TLS in G1/G2 NF-PanNETs was significantly greater than in PanNECs $(p=0.035$, online supplemental figure S2B). 

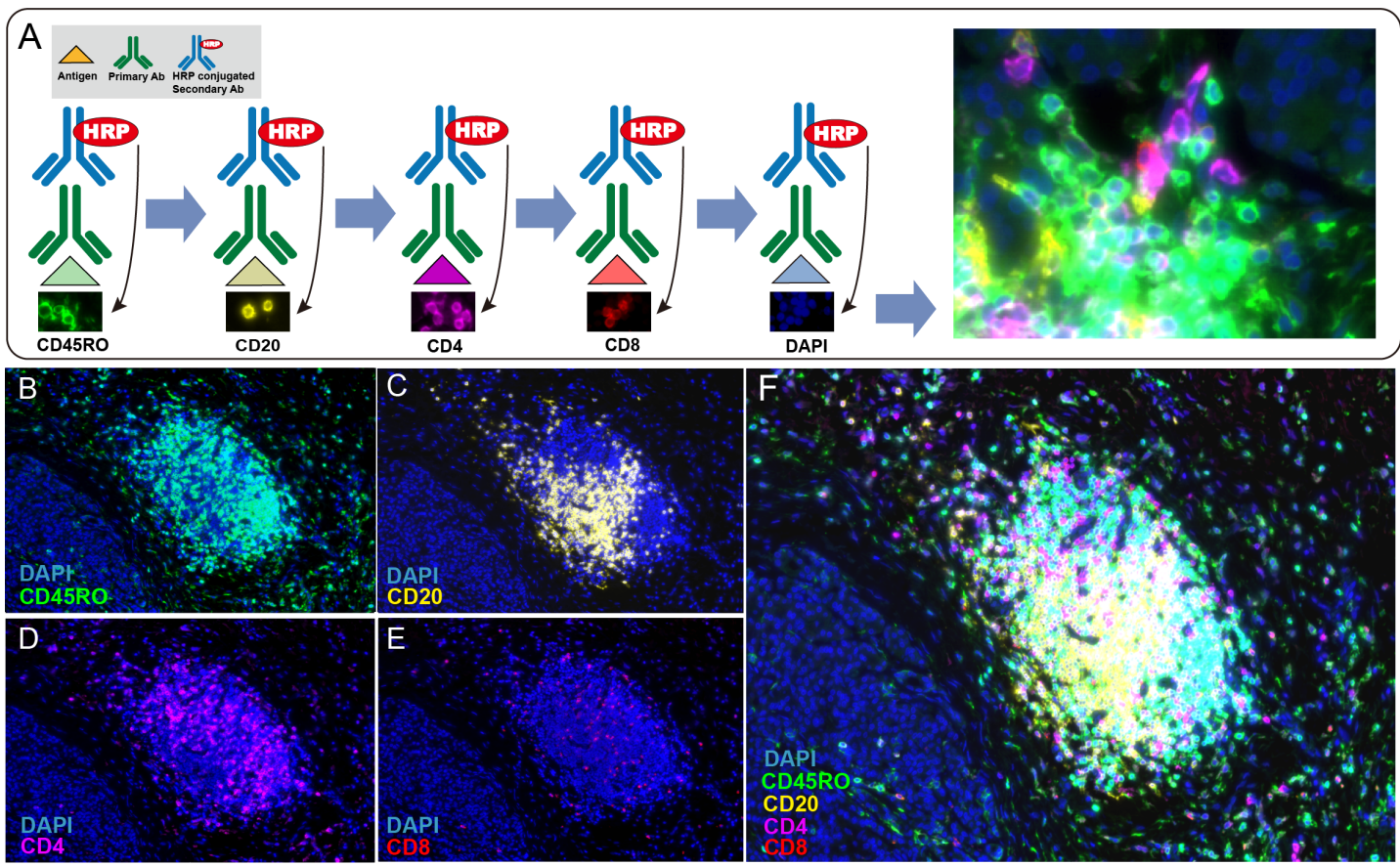

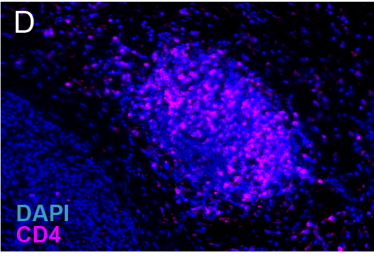

G

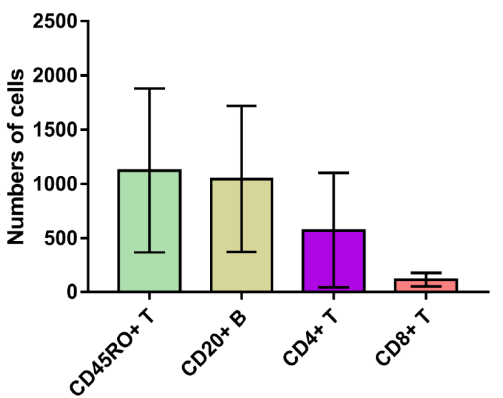

$\mathrm{H}$

Cellular composition of TLS

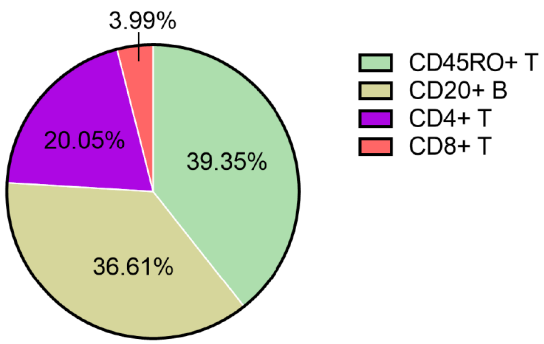

Figure 2 Multispectral fluorescent immunohistochemistry on G1/G2 non-functional pancreatic neuroendocrine tumors (NF-PanNETs). (A) A schematic of the sequential antibody staining approach used for multispectral immunohistochemistry indicating the color used for each protein of interest: CD45RO, green; CD20, yellow; CD4, violet; CD8, red. Cell nuclei were stained with DAPI (blue). (B-E) G1/G2 NF-PanNET tissues showing the localization of each of CD45RO, CD20, CD4, CD8. Magnification: 100×. (F) Merged image showing the colocalizations of DAPI, CD45RO, CD20, CD4 and CD8. Magnification: 100x. (G) Quantification of the numbers of cells positive for each of CD45RO, CD20, CD4, and CD8. The histogram displays the average number of each kind of cell counted in the five TLS shown in figure 2F and online supplemental figure S1. Error bars represent SD of the mean. $(H)$ Pie chart displaying the percentages of each kind of cell counted in (G) to describe the immune cell composition of the TLS. G1/G2, grade 1/grade 2; HRP, horseradish peroxidase; TLS, tertiary lymphoid structures.

\section{Relationship between tumor-infiltrating immune cells and TLS} Tumor-infiltrating $\mathrm{CD}^{+}$and $\mathrm{CD}^{+} \mathrm{T}$ cells, B cells, and

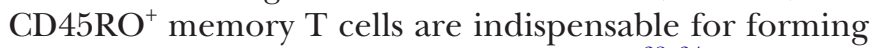
immune-responsive microenvironment, ${ }^{32-34}$ whereas TAMs are associated with immune-suppressive microenvironment. ${ }^{12}$ We identified significantly higher infiltration of $\mathrm{CD} 4^{+} \mathrm{T}$ cells in G1/G2 NF-PanNETs containing more than five TLS $\left(\mathrm{TLS}_{\geq 5}\right)$ compared with those having one to four TLS $\left(\mathrm{TLS}_{1-4}\right)$, or no TLS (TLS, $\mathrm{p}=0.031$ and $\mathrm{p}=0.011$, respectively). The numbers of tumor-infiltrating $\mathrm{CD}^{+} \mathrm{T}$ cells $(\mathrm{p}=0.032$ and $\mathrm{p}=0.014$, respectively) and CD20 $0^{+}$B cells $(p=0.031$ and $p<0.001$, respectively) were also significantly greater in tumor tissues with $\mathrm{TLS}_{1-4}$ and $\mathrm{TLS}_{>5}$ compared with $\mathrm{TLS}_{0}$. Additionally, the number of $\mathrm{CD} 45 \mathrm{RO}^{+}$memory $\mathrm{T}$ cells was significantly higher in TLS $_{\geq 5}$ G1/G2 NF-PanNETs than TLS 0 G1/G2 NF-PanNETs $(\mathrm{p}=0.048)$. In contrast, the number of $\mathrm{CD} 68^{+} \mathrm{TAMs}$ in TLS $_{1-4}$ and TLS $\mathrm{TLF}_{25}$ tumors was significantly lower than in $\mathrm{TLS}_{0}$ tumors $(\mathrm{p}=0.021$ and $\mathrm{p}=0.007$, respectively). Moreover, no significant differences in the numbers of tumorinfiltrating CD11 ${ }^{+}$DCs and NK cells were uncovered based on TLS number (online supplemental figure S3). Together, these data suggested that TLS might promote immune-responsive microenvironment.

\section{Association of the presence of TLS with survival of patients with G1/G2 NF-PanNETs}

We explored the association of the presence of TLS with RFS and OS in both the Fudan cohort and external validation set to determine whether TLS might be useful in determining patient prognosis. In the Fudan cohort, median RFS was 39 months (range, 1.5-95.0 months) and 
A

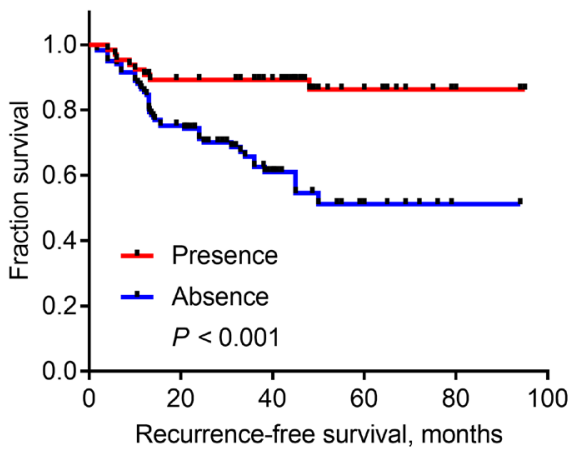

No. at risk

Presence

Absence

$\begin{array}{rrrrrr}65 & 56 & 47 & 15 & 3 & 0 \\ 117 & 85 & 31 & 8 & 1 & 0\end{array}$
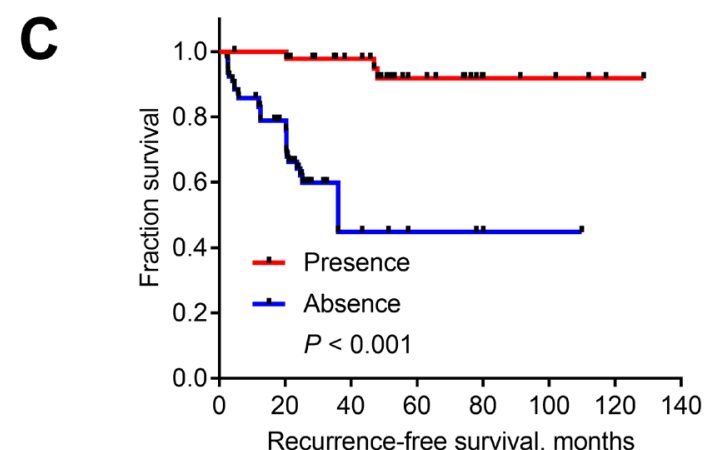

No. at risk

Presence

Absence

$\begin{array}{llllllll}46 & 45 & 35 & 20 & 10 & 8 & 2 & 0\end{array}$

B

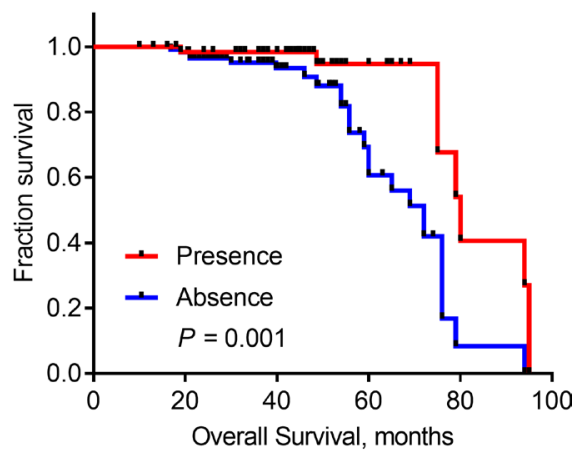

No. at risk

Presence

Absence

$\begin{array}{rrrrrr}65 & 59 & 48 & 15 & 3 & 0 \\ 117 & 112 & 49 & 14 & 1 & 0\end{array}$

D

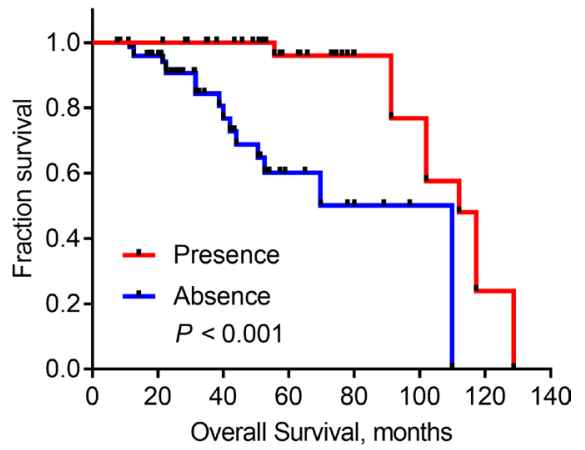

No. at risk

Presence

Absence

Figure 3 Kaplan-Meier curves depicting recurrence-free survival (RFS) and overall survival (OS) for patients with and without tertiary lymphoid structures (TLS). Association of the presence of TLS with RFS and OS in the Fudan cohort (A,B) and external validation set $(C, D)$.

median OS was 58 months (range, 10.0-96.0 months). The presence of TLS was associated with longer RFS and OS $(p<0.001$ and $p=0.001$, respectively, figure $3 A, B)$. In the external cohort, median RFS was 37 months (range, 2.3-128.7 months) and median OS was 56 months (range, 7.6-128.7 months). The presence of TLS was associated with longer RFS and OS $(p<0.001$ and $p<0.001$, respectively, figure 3C,D).

Next, we explored the correlation between the number of TLS and patient survival, and found that TLS number $\left(\mathrm{TLS}_{1-4}\right.$ vs $\left.\mathrm{TLS}_{\geq 5}\right)$ did not significantly impact either RFS or OS; however, patients with any number of TLS exhibited longer RFS and OS than those without TLS (online supplemental table $\mathrm{S} 1$ ).

Multivariate survival analyses indicated that $\mathrm{AJCC}^{8 \text { th }}$ TNM stage, WHO classification and the presence of TLS were independent prognostic factors of RFS ( $\mathrm{HR}=2.8$, 95\% CI: $1.79-4.27, \mathrm{p}<0.001$; HR $=2.9,95 \%$ CI: $1.57-5.22$, $\mathrm{p}=0.001$ and $\mathrm{HR}=0.3,95 \%$ CI: $0.14-0.69, \mathrm{p}=0.004$, respectively) and $\mathrm{OS}$ (HR=2.2, 95\% CI: 1.14-4.20, $\mathrm{p}=0.019$; $\mathrm{HR}=3.0,95 \%$ CI: $1.33-6.75, \mathrm{p}=0.008$ and $\mathrm{HR}=0.3,95 \%$ CI: $0.12-0.74, \mathrm{p}=0.009$, respectively) in the Fudan cohort. Likewise, these results were confirmed in the external validation set (table 2).

\section{Nomogram predicts the probability of 5-year RFS for resected G1/G2 NF-PanNETs}

Median follow-up time was 60 months, and there were 51 recurrences in the 182-patient Fudan cohort and 32 recurrences in the 125-patient external validation set. We aimed to construct a nomogram, incorporating the presence of TLS, to predict the probability of 5-year RFS, based on the multivariate Cox model (figure 4A). The nomogram c-index on the Fudan cohort was 0.772, superior to c-indices based on the WHO classification (0.665) and AJCC $^{\text {th }}$ TNM stage $(0.720)$. The nomogram c-index on the external validation set was 0.879 , which was also superior to that based on the WHO classification (0.777) and JJCC $^{\text {th }}$ TNM staging (0.807). The calibration plots for the Fudan cohort and external validation set showed good agreement between the predicted and observed 5-year recurrence-free probabilities (figure 4B,C).

Memorial Sloan-Kettering Cancer Center (MSKCC) constructed a nomogram using four variables: number of positive lymph nodes, Ki67 index, tumor diameter, and presence of vascular or perineural invasion. ${ }^{6}$ For comparison, we constructed a MSKCC-based nomogram using these same variables and applied the nomogram to the Fudan cohort and external validation set (figure 4D). ${ }^{6}$ 


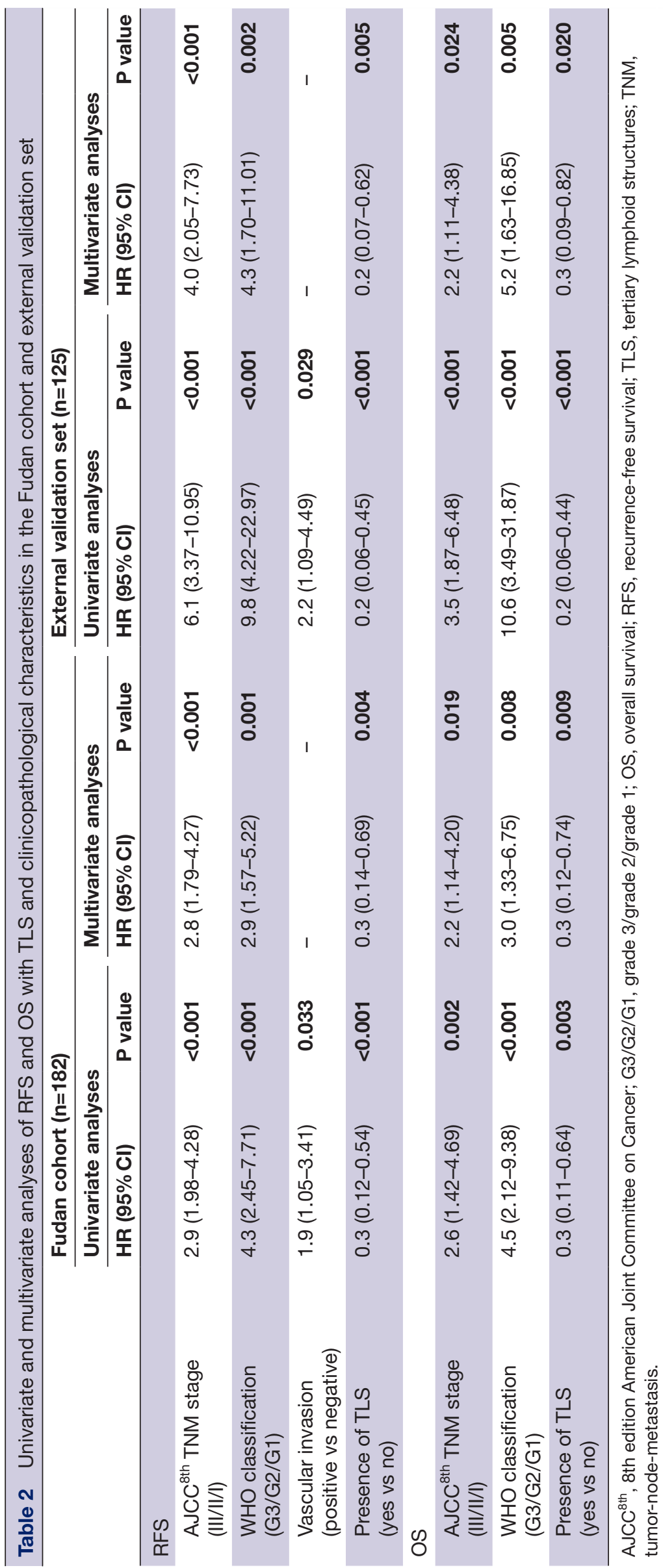




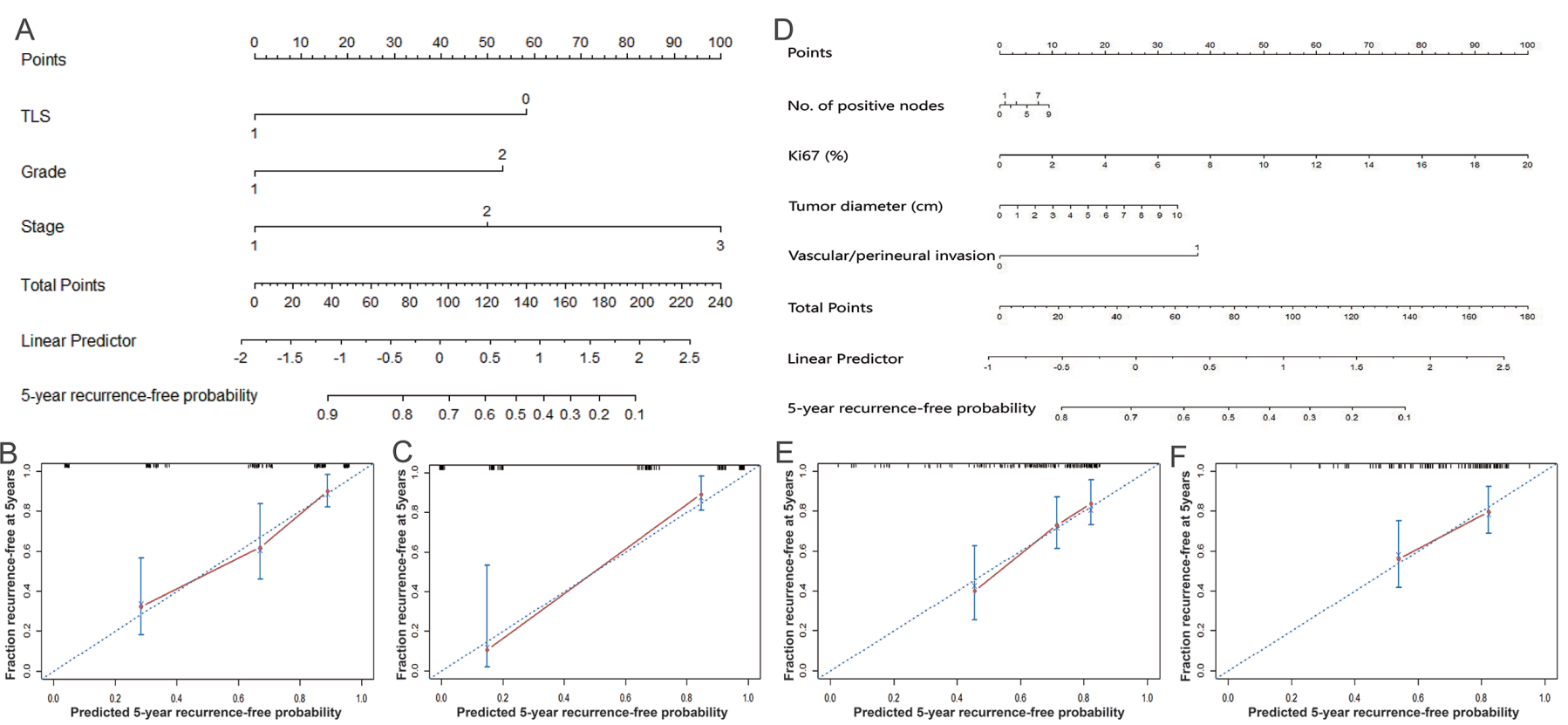

Figure 4 Nomogram predicting the probability of 5-year recurrence-free survival (RFS) and calibration plots. (A) A nomogram incorporating the presence of TLS, WHO classification and AJCC ${ }^{8 \text { th }}$ TNM stage. (B) Calibration curve for predicting the probability of 5-year RFS using the nomogram (A) on the Fudan cohort. (C) Calibration curve for predicting the probability of 5-year RFS using the nomogram (A) on the external validation set. (D) The MSKCC-based nomogram incorporating number of positive lymph nodes, Ki67 index, tumor diameter, and the presence of vascular or perineural invasion. (E) Calibration curve for predicting the probability of 5-year RFS by the MSKCC-based nomogram on the Fudan cohort. (F) Calibration curve for predicting the probability of 5-year RFS by the MSKCC-based nomogram on the external validation set. AJCC ${ }^{8 \text { th }}$, 8th edition American Joint Committee on Cancer; MSKCC, Memorial Sloan-Kettering Cancer Center; TLS, tertiary lymphoid structures; TNM, tumor-node-metastasis.

The c-indices of the MSKCC-based nomogram on the Fudan cohort and external validation set were 0.692 and 0.719 , respectively, indicating less discrimination than the new nomogram based on TLS, WHO classification and AJCC $^{\text {th }}$ TNM stage. Calibration plots for the two cohorts were shown in figure 4E,F.

\section{DISCUSSION}

To better define the implications of TLS in G1/G2 NF-PanNETs, we analyzed tumors from a 182-patient Fudan cohort and a 125-patient external validation set. Tumors were examined for the presence of TLS, and when present, for their cellular composition, association with tumor-infiltrating immune cells, to provide information on the tumor-immune microenvironment, and to understand their prognostic significance. Finally, we introduced a strategy to improve clinical prognostic accuracy.

To our knowledge, this is the first study to describe the presence and prognostic significance of TLS in G1/G2 NF-PanNETs. We identified TLS in tumors from $35.7 \%$ of enrolled patients. In NF-PanNETs, the TLS were located peritumorally, either just outside the tumor tissue or in the stromal area. Interestingly, the location of TLS in NF-PanNETs was distinct from that reported for patients with pancreatitis and pancreatic ductal adenocarcinoma (PDAC). ${ }^{35}$ In chronic pancreatitis and autoimmune pancreatitis, TLS were distributed evenly in pancreatic tissues. ${ }^{35}$ In PDAC, TLS were generally located peritumorally and adjacent to vessels (ie, venules and capillaries). ${ }^{18}$ Tumor tissues were typically surrounded by peritumoral TLS with only a few cases showing intratumoral TLS. Nonetheless, the rare presence of intratumoral TLS was an independent, favorable prognostic factor in PDAC. ${ }^{18}{ }^{36}$ The unique distribution of TLS in tumor/pancreatic tissues from patients with NF-PanNETs, pancreatitis, and PDAC, may reflect completely different immune microenvironment among these diseases. TLS were mainly composed of B-cell follicles with germinal centers and T-cell zones with DCs, having a distribution similar to SLO. Previous work showed that $\mathrm{CD} 4^{+} \mathrm{T}$ cells within TLS could support the differentiation of $\mathrm{CD} 88^{+} \mathrm{T}$ cells. ${ }^{37}$ DCs within TLS could present tumor antigens to the T cells located in the TLS, which could be critical for $\mathrm{T}$ cell-mediated anti-tumor responses. ${ }^{17}$ Consistent with Cai $e t a l,{ }^{38}$ we rarely detected FOXP3 $^{+}$Tregs in G1/G2 NF-PanNET tissues, though the presence of Tregs in TLS has been reported to be negatively associated with the activation of $\mathrm{CD}^{+}$and $\mathrm{CD} 8^{+} \mathrm{T}$ cells, inhibiting immune response against tumors. ${ }^{39}$ In order to clearly demonstrate the cellular composition of TLS, specifically relationship to cells of adaptive immune system, we conducted multispectral immunohistochemical analysis, which supported the results of our conventional IHC staining, but provided additional information regarding the quantity and spatial relationships between different populations of immune cells. 
We also showed that TLS were more likely to be detected in patients who had negative lymph node, absence of vascular and perineural invasion, lower TNM stage (I, II), and lower WHO grade (G1), although only absence of vascular invasion reached statistical significance. For better characterization of TLS in heterogeneous PanNENs, we examined other types of PanNENs, G1/G2 functional PanNETs, G3 NF-PanNETs, and PanNECs for the presence of TLS. Others have shown that the prognoses of patients with NF-PanNETs were poorer when compared to patients with functional PanNETs, especially for insulinoma, and were significantly better than patients with poorly differentiated PanNECs. ${ }^{40-42}$ We found that the percentage of TLS in G1/G2 NF-PanNETs was lower than in G1/G2 functional PanNETs, although this difference was not statistically significant. Additionally, as the tumor grade increases, the percentage of TLS decreases significantly. This supported TLS as a favorable prognostic indicator. Further, we demonstrated that immune cells derived from the adaptive immune system (ie, $\mathrm{CD}_{4}^{+} \mathrm{T}$ and $\mathrm{CD} 8^{+} \mathrm{T}$ cells, $\mathrm{CD} 20^{+} \mathrm{B}$ cells, and $\mathrm{CD} 45 \mathrm{RO}^{+}$memory $\mathrm{T}$ cells) were more abundant in tumor tissues with TLS than those without TLS. In contrast, the numbers of immune cells derived from the innate immune system (ie, CD68 ${ }^{+}$ TAMs) in G1/G2 NF-PanNET tissues containing TLS were significantly lower than those lacking TLS. These findings are similar to those of previous studies, ${ }^{183743}$ and indicate that TLS might allow for higher infiltration of effector immune cells, thus rendering a more effective anti-tumor immune response. Given the close correlation between the presence of TLS and the infiltration of adaptive immune cells into the tumors, TLS might serve as a potential biomarker of effective immunotherapies.

Univariate and multivariate survival analyses demonstrated that the presence of TLS in G1/G2 NF-PanNETs was an independent prognostic indicator of longer RFS and OS in both the Fudan cohort and external validation set. Surprisingly, we found the number of TLS did not impact RFS and OS. We used data from this study to construct a nomogram that accurately predicted the probability of 5-year RFS of resected G1/G2 NF-PanNETs, with better predictive ability than predictions based on WHO classification or $\mathrm{AJCC}^{8 \text { th }} \mathrm{TNM}$ stage. We propose that a nomogram incorporating the presence of TLS, WHO classification, and AJCC ${ }^{\text {sth }}$ TNM stage would improve personalized surveillance, facilitate the design of clinical trials, and potentially assist clinical practice.

In addition to the MSKCC nomogram, there are other prediction models of RFS in NF-PanNETs. However, these predictive models have one or more limitations, such as being derived from single-institution data, including only clinicopathological characteristics, and/or lacking validation with an external cohort. For example, Genc et $a t^{44}$ proposed a new scoring system to predict recurrence in G1/G2 NF-PanNETs that included tumor grade, positive lymph nodes, and perineural invasion, but lacked an external validation set. Similarly, Ellison $e t a t^{45}$ developed a simple prognostic tool using grade, sex, and binary age based on a single-institution series that lacked external validation. Our institution also generated a recurrent-risk stage system based on independent predictors of recurrence in PanNETs including $\mathrm{T}$ stage, $\mathrm{N}$ stage, insulinoma and Ki67 index. ${ }^{41}$ In contrast to these models, our newly constructed nomogram includes not only clinical characteristics but also immune microenvironment components-TLS, which might help us predict the prognosis of NF-PanNETs more accurately. Overall, our data indicate that our nomogram is as good, if not better than the aforementioned prognostic models of Genc, Ellison, and our own institution.

Accumulated data suggest that TLS may be important for eliciting strong cellular and humoral immune responses. For example, Lutz et $a t^{46}$ showed that TLS formed in immune-responsive patients with PDAC following GVAX (a granulocyte-macrophage colony-stimulating factor gene-modified tumor cell vaccine) treatment, whereas non-immune-responsive patients did not form TLS following GVAX treatment. Likewise, patients with highgrade cervical intraepithelial neoplasia, who received therapeutic administration of a human papillomavirus serotype 16 vaccine, formed TLS, whereas untreated patients did not. ${ }^{47}$ These findings might provide insights on the future research about NF-PanNETs and prompt in-depth studies on the role of TLS in tumor-immune microenvironment and immunotherapies.

Several limitations exist in the current study. First, all retrospective studies have inherent limitations. Second, given the retrospective nature of the study and the low prevalence of NF-PanNETs, we relied on IHC and mIHC to analyze samples for their cellular composition rather than flow cytometry, as the fresh samples required for flow cytometry were difficult to obtain. Additionally, this study did not investigate the association among tumor mutation background, immune checkpoints molecules (ie, PD-1 and its ligands PD-L1/PD-L2) and TLS. It will be important to define the composition of tumorimmune microenvironment and dissect TLS in NF-PanNETs in the future. Finally, prospective clinical trials will be required to investigate the possibility of using TLS as a biomarker for tumors that may be more responsive to immunotherapies.

Overall, the presence of TLS in G1/G2 NF-PanNET tissues appeared to be an independent prognostic factor of RFS and OS and was suggested to represent an immuneresponsive microenvironment.

\section{Author affiliations \\ ${ }^{1}$ Department of Pancreatic Surgery, Fudan University Shanghai Cancer Center, Shanghai, China \\ ${ }^{2}$ Department of Oncology, Shanghai Medical College, Fudan University, Shanghai, China \\ ${ }^{3}$ Shanghai Pancreatic Cancer Institute, Shanghai, China \\ ${ }^{4}$ Pancreatic Cancer Institute, Fudan University, Shanghai, China}

Acknowledgements The authors thank Professor Dan Huang (Department of Pathology, Fudan University Shanghai Cancer Center, Shanghai, China) and Professor Hai-Ying Zeng (Department of Pathology, Zhongshan Hospital, Fudan 
University, Shanghai, China) for their assistance in the pathological diagnosis of NF-PanNETs in all enrolled patients.

Contributors Wu-Hu Zhang, Wen-Quan Wang, Liang Liu and Xian-Jun Yu conceived the original idea of the study. Xuan Han, He-Li Gao, Shuai-Shuai Xu, and Tian-Jiao Li contributed to sample preparation and carried out the experiment. Wu-Hu Zhang, Shuo Li, Hua-Xiang Xu and Hao Li conducted all statistical analyses. Jiang Long, Long-Yun Ye, Xuan Lin and Chun-Tao Wu contributed to the implementation of the research and interpretation of data. All authors discussed the results, prepared, shaped the research and gave final approval of the manuscript to be published.

Funding This work was supported by grants from the National Science Foundation for Distinguished Young Scholars of China (81625016), the National Natural Science Foundation of China (81872366, 81871941, 81827807, 81802675, 81701630 and 81702341), the Outstanding Academic Leader Program of the 'Technological Innovation Action Plan' of Shanghai Science and Technology Commission (18XD1401200), the Scientific Innovation Project of Shanghai Education Committee (2019-01-07-00-07-E00057), the Natural Science Foundation of Shanghai (19ZR1410800), the Clinical and Scientific Innovation Project of Shanghai Hospital Development Center (SHDC12018109), and the Young Talented Specialist Training Program of Shanghai.

Competing interests There are no competing interests.

Patient consent for publication Not required.

Ethics approval This study was approved by the institutional research ethics committee of FUSCC. In accordance with the ethics committee's regulations, informed consent was obtained from patients included in this study.

Provenance and peer review Not commissioned; externally peer reviewed.

Data availability statement All data relevant to the study are included in the article or uploaded as supplemental information. All data analyzed in this study are included in this published article and its supplemental materials.

Supplemental material This content has been supplied by the author(s). It has not been vetted by BMJ Publishing Group Limited (BMJ) and may not have been peer-reviewed. Any opinions or recommendations discussed are solely those of the author(s) and are not endorsed by BMJ. BMJ disclaims all liability and responsibility arising from any reliance placed on the content. Where the content includes any translated material, BMJ does not warrant the accuracy and reliability of the translations (including but not limited to local regulations, clinical guidelines, terminology, drug names and drug dosages), and is not responsible for any error and/or omissions arising from translation and adaptation or otherwise.

Open access This is an open access article distributed in accordance with the Creative Commons Attribution Non Commercial (CC BY-NC 4.0) license, which permits others to distribute, remix, adapt, build upon this work non-commercially, and license their derivative works on different terms, provided the original work is properly cited, appropriate credit is given, any changes made indicated, and the use is non-commercial. See http://creativecommons.org/licenses/by-nc/4.0/.

ORCID iDs

Xian-Jun Yu http://orcid.org/0000-0002-6697-7143

Liang Liu http://orcid.org/0000-0002-8003-0503

\section{REFERENCES}

1 Amador Cano A, García F, Espinoza A, et al. Nonfunctional neuroendocrine tumor of the pancreas: case report and review of the literature. Int J Surg Case Rep 2013;4:225-8.

2 Cloyd JM, Poultsides GA. Non-functional neuroendocrine tumors of the pancreas: advances in diagnosis and management. World $J$ Gastroenterol 2015;21:9512-25.

3 Halfdanarson TR, Rubin J, Farnell MB, et al. Pancreatic endocrine neoplasms: epidemiology and prognosis of pancreatic endocrine tumors. Endocr Relat Cancer 2008;15:409-27.

4 Metz DC, Jensen RT. Gastrointestinal neuroendocrine tumors: pancreatic endocrine tumors. Gastroenterology 2008;135:1469-92.

5 Landoni L, Marchegiani G, Pollini T, et al. The evolution of surgical strategies for pancreatic neuroendocrine tumors (Pan-NENs): timetrend and outcome analysis from 587 consecutive resections at a high-volume institution. Ann Surg 2019;269:725-32.

6 Pulvirenti A, Javed AA, Landoni L, et al. Multi-institutional development and external validation of a nomogram to predict recurrence after curative resection of pancreatic neuroendocrine tumors. Ann Surg 2019. doi:10.1097/SLA.0000000000003579. [Epub ahead of print: 24 Sep 2019].

7 Pedraza-Arévalo S, Gahete MD, Alors-Pérez E, et al. Multilayered heterogeneity as an intrinsic hallmark of neuroendocrine tumors. Rev Endocr Metab Disord 2018;19:179-92.

8 Cives M, Pelle' E, Quaresmini D, et al. The tumor microenvironment in neuroendocrine tumors: biology and therapeutic implications. Neuroendocrinology 2019;109:83-99.

9 da Silva A, Bowden M, Zhang S, et al. Characterization of the neuroendocrine tumor immune microenvironment. Pancreas 2018;47:1123-9.

10 Takahashi D, Kojima M, Suzuki T, et al. Profiling the tumour immune microenvironment in pancreatic neuroendocrine neoplasms with multispectral imaging indicates distinct subpopulation characteristics concordant with who 2017 classification. Sci Rep 2018;8:13166.

11 Cives M, Strosberg J, Al Diffalha S, et al. Analysis of the immune landscape of small bowel neuroendocrine tumors. Endocr Relat Cancer 2019;26:119-30.

12 Zhang W-H, Wang W-Q, Gao H-L, et al. The tumor immune microenvironment in gastroenteropancreatic neuroendocrine neoplasms. Biochim Biophys Acta Rev Cancer 2019;1872:188311.

13 Sautès-Fridman C, Petitprez F, Calderaro J, et al. Tertiary lymphoid structures in the era of cancer immunotherapy. Nat Rev Cancer 2019;19:307-25.

14 Dieu-Nosjean M-C, Giraldo NA, Kaplon H, et al. Tertiary lymphoid structures, drivers of the anti-tumor responses in human cancers. Immunol Rev 2016;271:260-75.

15 Colbeck EJ, Ager A, Gallimore A, et al. Tertiary lymphoid structures in cancer: drivers of antitumor immunity, immunosuppression, or bystander sentinels in disease? Front Immunol 2017;8:8.

16 Engelhard VH, Rodriguez AB, Mauldin IS, et al. Immune cell infiltration and tertiary lymphoid structures as determinants of antitumor immunity. J Immunol 2018;200:432-42.

17 Goc J, Germain C, Vo-Bourgais TKD, et al. Dendritic cells in tumorassociated tertiary lymphoid structures signal a Th1 cytotoxic immune contexture and license the positive prognostic value of infiltrating CD8+ T cells. Cancer Res 2014;74:705-15.

18 Hiraoka N, Ino Y, Yamazaki-Itoh R, et al. Intratumoral tertiary lymphoid organ is a favourable prognosticator in patients with pancreatic cancer. Br J Cancer 2015;112:1782-90.

19 Hennequin $A$, Derangère $V$, Boidot $R$, et al. Tumor infiltration by Tbet+ effector T cells and CD20+ B cells is associated with survival in gastric cancer patients. Oncoimmunology 2016;5:e1054598.

20 Dieu-Nosjean M-C, Antoine M, Danel C, et al. Long-term survival for patients with non-small-cell lung cancer with intratumoral lymphoid structures. J Clin Oncol 2008;26:4410-7.

21 Martinet L, Garrido I, Filleron T, et al. Human solid tumors contain high endothelial venules: association with T- and B-lymphocyte infiltration and favorable prognosis in breast cancer. Cancer Res 2011;71:5678-87.

22 Lee HJ, Park IA, Song IH, et al. Tertiary lymphoid structures: prognostic significance and relationship with tumour-infiltrating lymphocytes in triple-negative breast cancer. J Clin Pathol 2016;69:422-30.

23 Finkin S, Yuan D, Stein I, et al. Ectopic lymphoid structures function as microniches for tumor progenitor cells in hepatocellular carcinoma. Nat Immunol 2015;16:1235-44.

24 Buisseret L, Desmedt C, Garaud S, et al. Reliability of tumorinfiltrating lymphocyte and tertiary lymphoid structure assessment in human breast cancer. Mod Pathol 2017;30:1204-12.

25 Coppola D, Nebozhyn M, Khalil F, et al. Unique ectopic lymph node-like structures present in human primary colorectal carcinoma are identified by immune gene array profiling. Am J Pathol 2011;179:37-45.

26 Wang W-Q, Liu L, Xu H-X, et al. Infiltrating immune cells and gene mutations in pancreatic ductal adenocarcinoma. Br J Surg 2016;103:1189-99.

27 Wang W-Q, Liu L, Xu H-X, et al. Intratumoral $\alpha$-SMA enhances the prognostic potency of CD34 associated with maintenance of microvessel integrity in hepatocellular carcinoma and pancreatic cancer. PLoS One 2013;8:e71189.

28 Zhang S-R, Yao L, Wang W-Q, et al. Tumor-infiltrating platelets predict postsurgical survival in patients with pancreatic ducta adenocarcinoma. Ann Surg Oncol 2018;25:3984-93.

29 Nghiem PT, Bhatia S, Lipson EJ, et al. PD-1 blockade with pembrolizumab in advanced Merkel-cell carcinoma. N Engl J Med 2016;374:2542-52.

30 Mezheyeuski A, Bergsland $\mathrm{CH}$, Backman M, et al. Multispectral imaging for quantitative and compartment-specific immune infiltrates reveals distinct immune profiles that classify lung cancer patients. J Pathol 2018;244:421-31. 
31 Harrell FE, Lee KL, Mark DB. Multivariable prognostic models: issues in developing models, evaluating assumptions and adequacy, and measuring and reducing errors. Stat Med 1996;15:361-87.

32 Hu G, Wang S. Tumor-infiltrating CD45RO ${ }^{+}$Memory T Lymphocytes Predict Favorable Clinical Outcome in Solid Tumors. Sci Rep 2017;7:10376.

33 Ino $\mathrm{Y}$, Yamazaki-Itoh $\mathrm{R}$, Shimada $\mathrm{K}$, et al. Immune cell infiltration as an indicator of the immune microenvironment of pancreatic cancer. Br J Cancer 2013;108:914-23.

34 Nelson $\mathrm{BH}$. CD20+ B cells: the other tumor-infiltrating lymphocytes. $\mathrm{J}$ Immunol 2010;185:4977-82.

35 Hiraoka N, Ino Y, Yamazaki-Itoh R. Tertiary lymphoid organs in cancer tissues. Front Immunol 2016;7:244.

36 Kuwabara S, Tsuchikawa T, Nakamura T, et al. Prognostic relevance of tertiary lymphoid organs following neoadjuvant chemoradiotherapy in pancreatic ductal adenocarcinoma. Cancer Sci 2019;110:1853-62.

37 de Chaisemartin L, Goc J, Damotte D, et al. Characterization of chemokines and adhesion molecules associated with $T$ cell presence in tertiary lymphoid structures in human lung cancer. Cancer Res 2011;71:6391-9.

38 Cai L, Michelakos T, Deshpande V, et al. Role of tumor-associated macrophages in the clinical course of pancreatic neuroendocrine tumors (PanNETs). Clin Cancer Res 2019;25:2644-55.

39 Joshi NS, Akama-Garren EH, Lu Y, et al. Regulatory T cells in tumorassociated tertiary lymphoid structures suppress anti-tumor $\mathrm{T}$ cell responses. Immunity 2015;43:579-90.
40 Yao JC, Hassan M, Phan A, et al. One hundred years after "carcinoid": epidemiology of and prognostic factors for neuroendocrine tumors in 35,825 cases in the United States. J Clin Oncol 2008;26:3063-72.

41 Gao H, Liu L, Wang W, et al. Novel recurrence risk stratification of resected pancreatic neuroendocrine tumor. Cancer Lett 2018;412:188-93.

42 Falconi M, Eriksson B, Kaltsas G, et al. ENETS consensus guidelines update for the management of patients with functional pancreatic neuroendocrine tumors and non-functional pancreatic neuroendocrine tumors. Neuroendocrinology 2016;103:153-71.

43 Sautès-Fridman C, Lawand M, Giraldo NA, et al. Tertiary lymphoid structures in cancers: prognostic value, regulation, and manipulation for therapeutic intervention. Front Immunol 2016;7:407.

44 Genç CG, Jilesen AP, Partelli S, et al. A new scoring system to predict recurrent disease in grade 1 and 2 nonfunctional pancreatic neuroendocrine tumors. Ann Surg 2018;267:1148-54.

45 Ellison TA, Wolfgang CL, Shi C, et al. A single institution's 26-year experience with nonfunctional pancreatic neuroendocrine tumors: a validation of current staging systems and a new prognostic nomogram. Ann Surg 2014;259:204-12.

46 Lutz ER, Wu AA, Bigelow E, et al. Immunotherapy converts nonimmunogenic pancreatic tumors into immunogenic foci of immune regulation. Cancer Immunol Res 2014;2:616-31.

47 Maldonado L, Teague JE, Morrow MP, et al. Intramuscular therapeutic vaccination targeting HPV16 induces T cell responses that localize in mucosal lesions. Sci Trans/ Med 2014;6:221ra13. 